\title{
The Importance of Radiopharmaceutical Chemistry for the Development of Nuclear Medicine: a Message from the Associate Editor
}

\author{
Jae Min Jeong ${ }^{1}$
}

Received: 10 July 2015 / Accepted: 13 July 2015 /Published online: 4 August 2015

(C) Korean Society of Nuclear Medicine 2015

It is common sense that radiopharmaceutical chemistry is extremely important for the development of nuclear medicine. As a radiopharmaceutical scientist, I strongly support Nuclear Medicine and Molecular Imaging (NMMI) to solicit and publish more papers about radiopharmaceuticals. Thus, it is a good opportunity for me to work as the Associate Editor of this journal to aid that purpose.

Historically, there were three key radionuclides: I-131, Tc99m and F-18. Those have led to outstanding progress being made in nuclear medicine. Recently, new radiopharmaceuticals such as Ga-68-labeled agents, Alzheimer disease imaging agents, liver cancer therapeutic agents, bone pain palliation alpha emitter, and Tc-99m-labeled mannose receptor imaging agent have been approved or enlisted in pharmacopeia. And many more, labeled with various radionuclides, are being studied extensively, which in the near future should lead to the next big jump forward in nuclear medicine since FDG.

NMMI was published as the Korean Journal of Nuclear Medicine from 1967 by the Korean Society of Nuclear Medicine. I remember that I published many manuscripts about radiopharmaceuticals in the KJNM written in Korean. Some of them are infancy, some of them make me remember how I struggled to write papers when I was young. The journal name KJNM was changed to NMMI in 2006 and was published in the Korean language by the KSNM for 5 years. KSNM changed NMMI into an English language journal, and commissioned its production and distribution by Springer in 2010, which made the journal truly international.

Our new Editor-in-Chief, Prof. Dong Soo Lee, reorganized the editors, and solicited myself, a radiopharmaceutical chemist, and Prof. Jae Sung Lee, a physicist, as the Associate Editors to emphasize the importance of basic sciences. I think it is a very timely measure for the promotion of the journal. The Journal of Nuclear Medicine, the most widely read journal in the nuclear medicine field, publishes about $40 \%$ of basic science articles, which might be the strong reason for the JNM to consistently have many readers and record a high impact factor. Its impact factor in 2014 was 6.160! This suggests that NMMI should publish more basic science papers.

I might have a role in soliciting good manuscripts about radiopharmaceutical sciences as the president-elect of the Society of Radiopharmaceutical Sciences (SRS) and the president of the Korean Society of Radiopharmaceuticals and Molecular Probes (KSRAMP). The development of a highquality nuclear medicine journal would be helpful for the radiopharmaceutical scientists.

Finally, I hope NMMI will develop into one of the most widely read nuclear medicine journals in the world with the support of members of SRS and KSRAMP.
Jae Min Jeong

jmjng@snu.ac.kr

1 Department of Nuclear Medicine, Seoul National University College of Medicine, Seoul, Korea 